\title{
Insulin und sein Rezeptor - Spezifität durch Kombinatorik?
}

\author{
THERESIA GUTMANN, ÜNAL COSKUN \\ PAUL-LANGERHANS-INSTITUT DRESDEN DES HELMHOLTZ-ZENTRUMS MÜNCHEN \\ AM UNIVERSITÄTSKLINIKUM CARL GUSTAV CARUS DER TU DRESDEN; DEUTSCHES \\ ZENTRUM FÜR DIABETESFORSCHUNG E. V. (DZD), NEUHERBERG
}

\section{Glucose homeostasis and growth essentially depend on insulin engag- ing its receptor. Combining biochemistry, structural biology, and molecular dynamics simulations, we demonstrated how insulin binding stabilizes the insulin receptor in a T-shaped conformation with up to four bound ligands: two at receptor site 1 and - for the first time - two at site 2. These findings expand the current models of insulin binding to its receptor and potentially inform new approaches to struc- ture-guided drug design.}

DOI: $10.1007 / \mathrm{s} 12268-020-1395-1$

(C) Die Autoren 2020

Membrangebundene Rezeptoren sind in der Regel keine Schalterproteine, die schlichtweg zwischen "An“- und „Aus“Zuständen wechseln. Stattdessen nehmen sie eine Vielzahl von Transitionszuständen an, deren Dynamik darüber hinaus durch Phosphatasen und akzessorische Proteine sowie durch die Lipidumgebung moduliert wird. Es mag daher nicht verwundern, dass das Peptidhormon Insulin nach der Bindung am Insulinrezeptor eine Vielzahl weitreichender Vorgänge auslöst, etwa die Glucoseaufnahme, Lipid- und Proteinbiosynthese, aber auch Zellteilung. Insulinrezeptoren findet man auf der Zelloberfläche beinahe jeder Körperzelle. Insulinbindung an der extrazellulären Domäne (Ektodomäne) führt zur Erhöhung der Autophosphorylierung der intrazellulären Kinasedomänen und unmittelbar zur Signalweiterleitung in das Zellinnere. Trotz jahrzehntelanger Forschung ist der Prozess der Insulinbindung am Rezeptor und dessen Signalweiterleitung unvollständig verstanden.

\section{Visualisierung des aktivierten Insulinrezeptors}

Die Aktivierung des Volllängen-Insulinrezeptors in einer Membranumgebung konnten wir erstmals direkt elektronenmikroskopisch visualisieren (Abb. 1). Unter basalen
Bedingungen ähnelt die Rezeptor-Ektodomäne einer umgedrehten „U“-Form, die nach Insulinbindung eine T-Form annimmt. Diese Konformationsänderung wird an die Transmembrandomänen übertragen, sodass diese sich annähern und infolgedessen die Autophosphorylierung der intrazellulären Kinasedomänen erleichtern [1, 2]. Demnach beruht die Signalübertragung auf der Kontrolle des Abstands zwischen den beiden Transmembrandomänen, welcher durch die Ektodomäne reguliert wird. Interessanterweise weisen die insulingebundenen T-ähnlichen Strukturen eine hohe strukturelle Heterogenität auf, wobei der horizontale Balken des „T“ mehr oder weniger zur Seite geneigt ist. Diese strukturelle Vielfalt weist auf verschiedene Übergangszustände hin, die möglicherweise mit unterschiedlicher Ligandenbesetzung zusammenhängen.

Wie genau interagiert Insulin mit seinem Rezeptor?

Obwohl T-ähnliche Strukturen von löslichen Ektodomänen im Komplex mit einem oder zwei Insulinen zwischenzeitlich von zwei anderen Gruppen unabhängig voneinander mittels Kryo-Elektronenmikroskopie (KryoEM) bestätigt wurden [3, 4], blieb eine der wichtigsten Fragen weiterhin unbeantwortet.

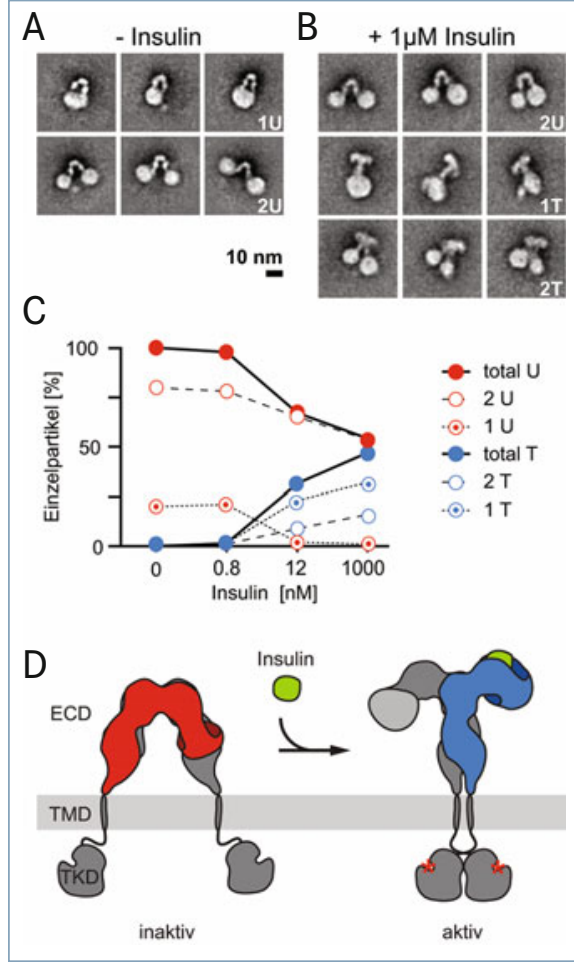

Abb. 1: Visualisierung der Insulin-induzierten Transmembran-Signalweiterleitung im Vollängen-Insulinrezeptor. Repräsentative elektronenmikroskopische 2D-Ansichten nach Einzelpartikelanalyse der in LipidNanodiscs eingebetteten Insulinrezeptoren. Nanodiscs sind ca. zwölf Nanometer große Lipiddoppelschicht-Scheibchen und hier als zirkuläre Strukturen erkennbar. A, In Abwesenheit von Insulin ähneln die Ektodomänen einem umgekehrten „U“. Beide Transmembrandomänen inserieren dabei entweder in einer gemeinsamen Disc (1U) oder in zwei separaten Discs (2U). B, Insulinbindung induziert eine Konformationsänderung in der Insulinrezeptor-Ektodomäne zu einer „T“-ähnlichen Struktur. C, Quantifizierung der Insulinrezeptorpopulationen mit einer speziellen Ektodomänenkonformation nach Zugabe der gegebenen Insulinkonzentrationen. D, Modell der Insulinrezeptoraktivierung. Ligandenbindung induziert Konformationsänderungen in der Ektodomäne (ECD), welche an die Transmembrandomänen (TMDs) gekoppelt sind. Das Zusammenführen der TMDs erleichtert die Interaktion der Tyrosinkinasedomänen (TKDs), was zur Erhöhung der Autophosphorylierung (rote Sternchen) führt (@ Gutmann et al. 2018 [1]). 
Basierend auf biochemischen und genetischen Studien am Liganden wurden bereits vor Jahrzehnten zwei diskrete Bindestellen (Site 1 und Site 2) im Insulinmolekül vermutet und konkrete Aminosäurereste für beide Sites identifiziert [5]. Beide Kryo-EMStudien bestätigten in der Tat Site 1-Interaktionen, erfassten aber die Site 2-Interaktionen nicht. Aus diesem Grund konzentrierten wir uns auf Strukturstudien der isolierten Ektodomäne [6].

\section{Strukturelle Aufklärung der Site 2}

Zunächst exprimierten und reinigten wir die vollständig glykosylierte humane Ektodomäne rekombinant aus humanen Zellen. Nach umfassender biochemischer Charakterisierung wurde das Protein für die Kryo-EM in Zusammenarbeit mit Ingmar Schäfer (Max-Planck-Institut für Biochemie, Martinsried) und Mike Strauß (McGill University, Montreal) genutzt (Abb. 2). Um alle Bindungsstellen zu sättigen, setzten wir für die elektronenmikroskopische Analyse die Rezeptor-Ektodomäne einem Überschuss an Insulin aus. Die 3D-Rekonstruktion der kompletten und unmodifizierten Ektodomäne bestätigte die Bindung von zwei Insulinen in der Kopfregion (die wir als Insuline 1 und $1^{\text {* }}$ bezeichnen). Zusätzlich konnten wir nun erstmals strukturell die Bindung von zwei weiteren Insulinen in der Stielregion nachweisen - Insulin 2 und 2'.

Parallel zur Kryo-EM verfolgten wir in Kollaboration mit Ilpo Vattulainen (Universität Helsinki) die Dynamik der insulinge-

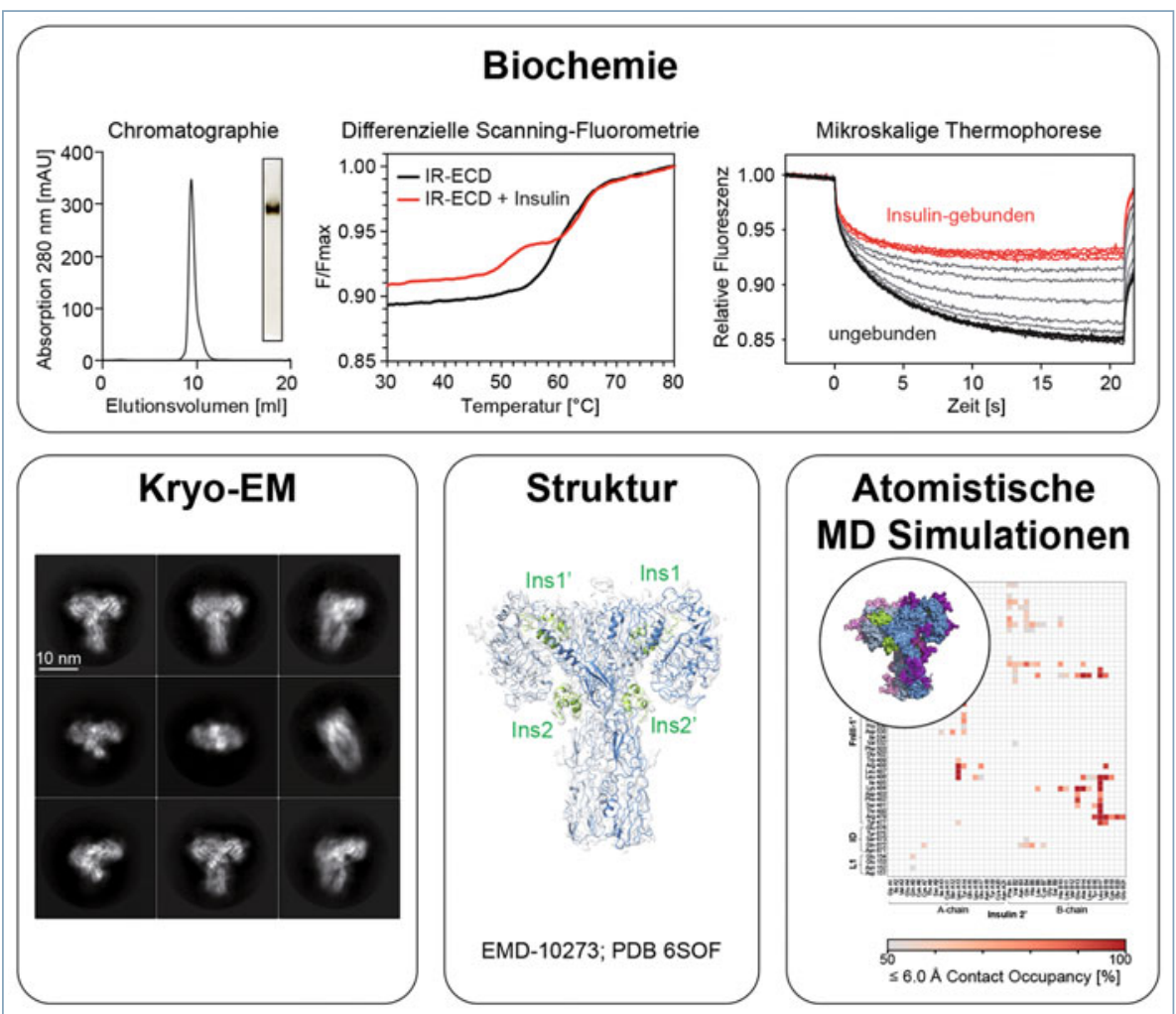

A Abb. 2: Kombinierter Ansatz zum Verständnis der Insulin-Insulinrezeptor-Interaktionen. Die gereinigte Insulinrezeptor-Ektodomäne wurde umfassend biochemisch charakterisiert. Insulinbindung an der löslichen Ektodomäne lässt sich mittels differenzieller Scanning-Fluorimetrie unter Nutzung der intrinsischen Tryptophanfluoreszenz - und damit völlig labelfrei - in einem Temperaturgradienten (nanoDSF) nachweisen. Bei Insulinbindung senkt sich die erste der beiden Schmelztemperaturen $\left(T_{m 1}\right)$ deutlich von 58,9 auf $51,1^{\circ} \mathrm{C}$. Mikroskalige Thermophorese lässt sich nutzen, um die Equilibrium-Dissoziationskonstante der Ektodomäne $\left(K_{\mathrm{d}} \approx 3 \times 10^{-8} \mathrm{M}\right)$ zu bestimmen, wobei auf eine Immobilisierung des Proteins oder gar eine Markierung des Liganden verzichtet werden kann. Kryo-Elektronenmikroskopie (Kryo-EM) wurde zur Strukturaufklärung der Insulin-gesättigten Ektodomäne genutzt (EMD-10273, PDB: 6SOF). Die pseudosymmetrische, aus zwei identischen kovalent verbundenen Protomeren bestehende Insulinrezeptor-Ektodomäne (blau) nimmt nach Insulinbindung eine „“-ähnliche Konformation ein, wobei bis zu vier Insuline (grün) an den Sites 1 bzw. 1'sowie 2 bzw. 2 'binden können [6]. Zwischenzeitlich konnte diese Struktur mit vier gebundenen Insulinen unabhängig bestätigt werden [7]. Parallel wurden die Insulin-InsulinrezeptorInteraktionen mithilfe atomistischer Molekulardynamik(MD)-Simulationen der glykosylierten Ektodomäne charakterisiert. ECD: Ektodomäne (@) Gutmann et al. 2019 [6]).

bundenen und komplett glykosylierten Ektodomäne mittels atomistischer Molekulardynamik(MD)-Simulationen. Die am Stiel gebundenen Insuline nutzen tatsächlich diejenigen Aminosäuren für die Interaktionen mit dem Rezeptor, welche bereits biochemisch als „Site 2“ vorhergesagt wurden. Interessanterweise weisen die Insuline in den Bindungstaschen 1 und 2 unterschiedliche Konformationen auf (Abb. 3). Die InsulinInsulinrezeptor-Interaktionen beruhen damit auf substanziellen Konformationsänderungen sowohl im Rezeptor als auch im Liganden selbst. Bei den Insulinen 1 und 1' steht die C-terminale B-Kette vom ansonsten kompakten Insulinmolekül ab, was als „offene Konformation“ bezeichnet wird und für eine hochaffine Bindung unerlässlich ist [8]. Dagegen haben die Insuline 2 und 2‘ eine „geschlossene Konformation“ - also jene, die der Konformation von ungebundenem Insulin in Lösung entspricht. Seitenketten, die maßgeblich an Site 1-Interaktionen beteiligt sind, sind im ungebundenen Insulinmolekül wie auch im Rezeptor teilweise unzugänglich. Im Gegensatz dazu sind die Seitenketten des Site 2-Insulins und des Rezeptors in beiden Konformationen exponiert.

Die Insulinbindung am Stiel ist damit sehr wahrscheinlich für die ersten Kontakte mit dem Rezeptor von Bedeutung. Es ist anzunehmen, dass Interaktionen an der Site 2 (bzw. 2‘) zumindest für die initialen, wenngleich transienten Liganden-Rezeptor-Kontakte wichtig sein könnten und möglicherweise zur Ligandenspezifität beitragen.

Die mit vier Insulinen gesättigte Rezeptorkonformation ist nicht zwangsläufig die physiologisch bedeutsamste im menschlichen Körper, aber sie erscheint als ein möglicher Zustand, bei dem alle, gegebenenfalls auch transienten, verfügbaren Bindungsstellen inklusive der Site 2 besetzt sind. Die VierInsulin-Konformation könnte z. B. bei lokal hohen Insulinkonzentrationen bzw. hohen Ligand/Rezeptor-Verhältnissen eine bedeutsame Rolle spielen, etwa in pankreatischen Inselzellen unmittelbar nach der Insulinsekretion.

\section{Ausblick}

Die Tatsache, dass die Insulinersatztherapie noch nicht das gesamte Spektrum der physiologischen Insulinwirkung rekapituliert, verdeutlicht, dass wir dringend ein umfassendes Verständnis des Prozesses der Insulinrezeptoraktivierung benötigen. Unsere Kryo-EM-Struktur und MD-Simulationen der 


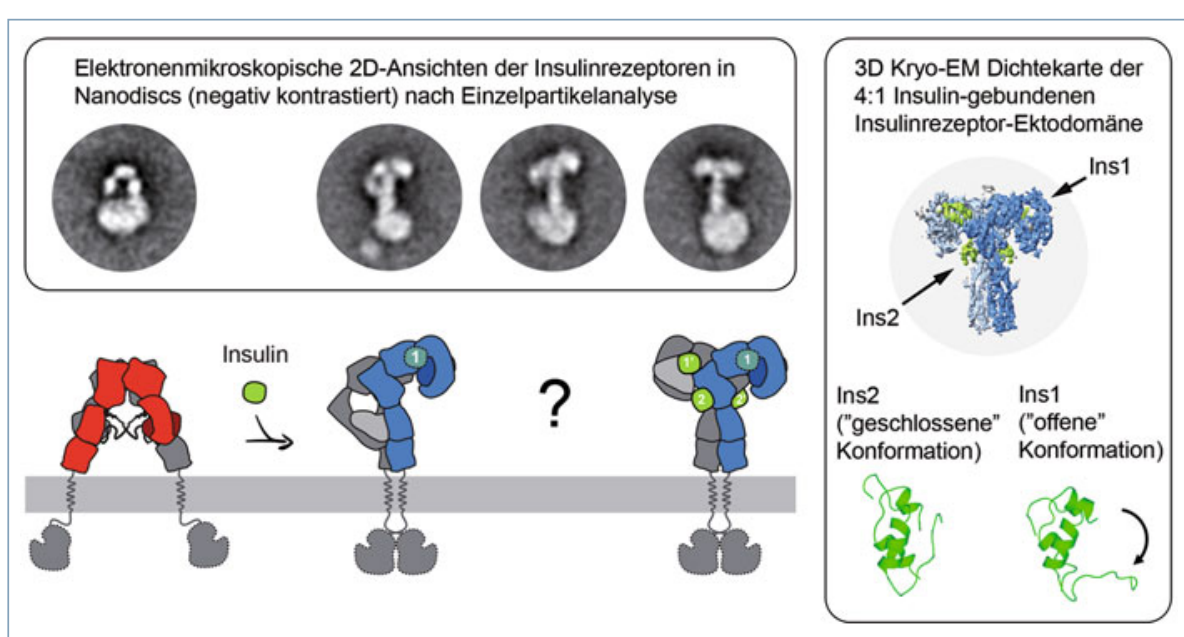

A Abb. 3: Schematische Illustration der ungebundenen und der insulingebundenen Transitionszustände, für welche Strukturen der kompletten Insulinrezeptor-Ektodomäne verfügbar sind, in Gegenüberstellung zu diversen Konformationen (oben), die wir elektronenmikroskopisch für den kompletten Insulinrezeptor in Lipid-Nanodiscs beobachten konnten [1]. Die Insulin-gesättigte Konformation konnten wir des Weiteren mittels Kryo-EM auflösen (rechts), wobei sich zeigt, dass Site 2/2'-gebundene Insuline eine geschlossene Konformation und Site 1/1'-gebundene Insuline eine „offene“ Konformation aufweisen (๔ Gutmann et al. 2019 [6]).

menschlichen Insulinrezeptor-Ektodomäne bieten neue Einblicke in die komplexen Insulin-Insulinrezeptor-Interaktionen und erlauben neue Interpretationen. Zunächst geben unsere Daten einen Hinweis darauf, wie Ligandenspezifität und Selektivität im zellulären Kontext umgesetzt werden könnten. Eine herausragende Fragestellung ist, wie die verschiedenen Liganden des Insulinrezeptors zu unterschiedlichen und kontrollierten Signalkaskaden führen können. Beim Menschen sind die relevanten Liganden des Insulinrezeptors neben Insulin die Insulinähnlichen Wachstumsfaktoren 1 und 2 (KF 1/2). Weitere Beispiele für die Ligandenheterogenität sind die homologen Insulinähnlichen Rezeptoren von Wirbellosen. Drosophila melanogaster exprimiert sieben Insulin-ähnliche Peptidliganden für einen einzigen Insulin-ähnlichen Rezeptor, während Caenorhabditis elegans sogar 40 Insulinähnliche Peptide besitzt. Diese Vielfalt unterstreicht, wie wichtig eine sorgfältige Untersuchung der selbst subtilsten Asymmetrien innerhalb der Rezeptoren ist, da diese unmittelbare Konsequenzen für die Affinität, Kooperativität und Ligandenselektivität haben können und somit in den unterschiedlichen zellulären Signalwegen von Bedeutung sein dürften.

Um zusätzliche Übergangszustände des Insulinrezeptors zu erfassen - idealerweise im Kontext des Vollängenrezeptors in seiner Membranumgebung -, sind weitere strukturelle Studien erforderlich. Parallel zu zellba- sierten Ansätzen könnten so bestimmte Konformationen mit spezifischen zellulären Reaktionen kausal in Beziehung gesetzt werden. Dafür haben wir eine experimentelle Pipeline etabliert, die es erlaubt, die Bindung und Struktur der Insulin-Rezeptor-Komplexe zu untersuchen, und die zudem für die Untersuchung neuartiger intelligenter Insuline oder Antagonisten geeignet ist. Zusätzlich bietet unser Molekulardynamik-Simulationssystem eine wertvolle Ressource für die Entwicklung und das Testen neuartiger Liganden in silico. Damit ist zu hoffen, dass wir so dem Ziel näherkommen, die Aktivierung von Insulinrezeptoren umfassend zu verstehen, um letztendlich einen Beitrag zum Verständnis der Rezeptorbiochemie und zur Verbes- serung des pharmakologischen Targetings zu leisten und somit unerwünschte Nebeneffekte, wie unkontrolliertes Zellwachstum, zu verhindern.

\section{Literatur}

[1] Gutmann T, Kim KH, Grzybek M et al. (2018) Visualization of ligand-induced transmembrane signaling in the fulllength human insulin receptor. J Cell Biol 217:1643-1649 [2] Gutmann T (2019) Der Insulinrezeptor in Aktion - wie Insulin seinen Rezeptor aktiviert. BIOspektrum 25:485-487 [3] Scapin G, Dandey VP, Zhang Z et al. (2018) Structure of the insulin receptor-insulin complex by single-particle cryoEM analysis. Nature 556:122-125

[4] Weis F, Menting JG, Margetts MB et al. (2018) The signalling conformation of the insulin receptor ectodomain. Nat Commun 9:4420

[5] De Meyts P (2015) Insulin/receptor binding: the last piece of the puzzle? What recent progress on the structure of the insulin/receptor complex tells us (or not) about negative cooperativity and activation. Bioessays 37:389-397 [6] Gutmann T, Schäfer IB, Poojari C et al. (2019) Cryo-EM structure of the complete and ligand-saturated insulin receptor ectodomain. J Cell Biol 219:e201907210

[7] Uchikawa E, Choi E, Shang G et al. (2019) Activation mechanism of the insulin receptor revealed by cryo-EM structure of the fully liganded receptor-ligand complex. Elife 8:e48630

[8] Hua QX, Shoelson SE, Kochoyan M et al. (1991) Receptor binding redefined by a structural switch in a mutant human insulin. Nature 354:238-241

\section{Funding: Open Access funding provided by Projekt DEAL.}

Open Access: Dieser Artikel wird unter der Creative Commons Namensnennun 4. International Lizenz veröffentlicht, welche die Nutzung, Vervielfältigung, Bearbeitung, Verbreitung und Wiedergabe in jeglichem Medium und Form erlaubt, sofern Sie den/die ursprünglichen Autor(en) und die Quelle
ordnungsgemäß nennen, einen Link zur Creative Commons Lizenz beifügen und ordnungsgemäß nennen, einen Link zur Creative Commons Lizenz beifu
angeben, ob Änderungen vorgenommen wurden. Die in diesem Artikel angeben, ob Änderungen vorgenommen wurden. Die in diesem Artikel
enthaltenen Bilder und sonstiges Drittmaterial unterliegen ebenfalls der genannten Creative Commons Lizenz, sofern sich aus der Abbildungslegende nichts anderes ergibt. Sofern das betreffende Material nicht unter der genannten Creative Commons Lizenz steht und die betreffende Handlung nicht nach gesetzlichen Vorschriften erlaubt ist, ist für die oben aufgeführten Weiterverwendungen des Materials die Einwilligung des jeweiligen Rechteinhabers einzuholen. Weitere Detalls zur Lizenz entnehmen Sie bitte der Lizenzinformation auf http://creativecommons.org/licenses/by/4.0/deed.de.

\section{Korrespondenzadresse:}

Dr. Ünal Coskun

Paul-Langerhans-Institut Dresden des Helmholtz Zentrums München am Universitätsklinikum

Carl Gustav Carus der TU Dresden

Fetscherstraße 74

D-01307 Dresden

coskun@plid.de 